\title{
Do all patients with breast cancer benefit from neoadjuvant chemotherapy?
}

\author{
Kyoung Eun Lee
}

Division of HematologyOncology, Department of Internal Medicine, Ewha Womans University School of Medicine, Seoul, Korea
Received: October 9, 2020

Accepted: October 16, 2020

\author{
Correspondence to \\ Kyoung Eun Lee, M.D. \\ Division of Hematology- \\ Oncology, Department of \\ Internal Medicine, Ewha \\ Womans University Mokdong \\ Hospital, 1071 Anyangcheon-ro, \\ Yangcheon-gu, Seoul 07985, \\ Korea \\ Tel: +82-2-2650-5030 \\ Fax: +82-2-2650-5062 \\ E-mail: oncolee@ewha.ac.kr \\ https://orcid.org/0000-0003- \\ 1596-8666
}

\section{See Article on Page 1489-1496}

Neoadjuvant chemotherapy (NAC) has been used for down-staging of locally advanced breast cancer since the 1970s, and early breast cancers (including locally advanced tumors) can also be good candidates for NAC. NAC can reduce tumor size, control early microscopic disease, and enable investigations of tumor biomarkers and immediate endpoints, among other benefits. However, the procedure delays curative resection in some patients. A meta-analysis of approximately 4,000 patients with breast cancer showed that NAC was equivalent to adjuvant therapy in terms of survival and disease progression. In addition, the National Surgical Adjuvant Breast and Bowel Project B-18 study revealed no differences in disease-free and overall survival between pre- and postoperative systemic chemotherapy [1-3]. The Collaborative Trials in Neoadjuvant Breast Cancer pooled analyses showed that a pathologic complete response (pCR) was associated with long-term outcomes, and a high pCR rate was obtained in HER2-positive breast cancer patients with trastuzumab and in triple negative breast cancer patients [4]. The pCR rate was not validated as a surrogate endpoint for improved survival outcomes but had adequate prognostic power.
Selection of an NAC regimen depends on the biologic subgroup of breast cancer. In the most recent guidelines, sequential anthracyclines and taxanes is the preferred NAC regimen; their concurrent administration may be feasible for HER2-negative early breast cancer [5,6]. HER2-positive tumors typically require an NAC regimen comprising one or two anti-HER2 agents [7].

In this issue of the Korean Journal of Internal Medicine, Won et al. [8] reports the clinical outcomes and factors predictive of efficacy for simultaneous docetaxel and epirubicin (DE) NAC in patients with locally advanced breast cancer. In their study, the down-staging rate for locally advanced breast cancer was $62.5 \%$, suggesting that change in the Ki67 level is a predictive factor. In two previous studies in the setting of treatment with combinations of anthracyclines and docetaxel, the clinical response and pCR rates were $68 \%-93 \%$ and $8 \%-16 \%$, respectively $[9,10]$. Unfortunately, in Won et al. [8], the pCR rate was lower than in previous studies, possibly because of a low number of treatment cycles, reduced efficacy, or inappropriate patient selection. NAC regimens comprising anthracyclines and taxanes can increase the incidence of febrile neutropenia, possibly delaying treatment or reducing its efficacy, although Won et al. [8] did not mention episodes of myelosuppres- 
sion. The authors also did not identify patient subgroups whose pCR rate would be increased by NAC. Although $27.5 \%$ of the patients were positive for HER2, they did not receive anti-HER2 therapy and only 10 patients $(25.0 \%)$ had triple-negative breast cancer, possibly explaining the low pCR rates in these patients.

Anthracyclines and taxanes are typically included in NAC regimens for HER2-negative early breast cancer. Indeed, the anthracyclines doxorubicin and epirubicin are the key chemotherapeutics for early breast cancer. Epirubicin is an epimer of doxorubicin and since the 1980 s has been used outside of the United States. In the United States, however, epirubicin did not receive marketing approval from the Food and Drug Administration until 1999. The efficacy of epirubicin is similar to that of doxorubicin but has a more favorable toxicity profile, which includes cardiac and hematological toxicities [11]. This retrospective study is the first in South Korea to focus on NAC with DE. The DE regimen, although not included in the National Comprehensive Cancer Network guidelines for HER2-negative breast cancers, could be an option for locally advanced breast cancer.

Most patients positive for HER2 receive anti-HER2 agents, including trastuzumab and/or pertuzumab, in the neoadjuvant/adjuvant setting, and some patients received NAC with prophylactic granulocyte colony-stimulating factor. These additions can enhance the efficacy of the DE regimen. Patients with HER2-positive breast cancer should receive anti-HER2 NAC but those positive for HR are unlikely to benefit. Further studies are required to determine the most effective combinations and sequences of drugs for the various biologic subgroups of breast cancer. In addition, novel biomarkers that enable prediction of the pCR and the long-term outcomes, as well as novel adjuvant therapies, are needed.

\section{Conflict of interest}

No potential conflict of interest relevant to this article was reported.

\section{REFERENCES}

1. Wolmark N, Wang J, Mamounas E, Bryant J, Fisher B. Preoperative chemotherapy in patients with operable breast cancer: nine-year results from National Surgical Adjuvant Breast and Bowel Project B-18. J Natl Cancer Inst Monogr 2001;30:96-102.

2. Rastogi P, Anderson SJ, Bear HD, et al. Preoperative chemotherapy: updates of National Surgical Adjuvant Breast and Bowel Project Protocols B-18 and B-27. J Clin Oncol 2008;26:778-785.

3. Mauri D, Pavlidis N, Ioannidis JP. Neoadjuvant versus adjuvant systemic treatment in breast cancer: a meta-analysis. J Natl Cancer Inst 2005;97:188-194.

4. Cortazar P, Zhang L, Untch M, et al. Pathological complete response and long-term clinical benefit in breast cancer: the CTNeoBC pooled analysis. Lancet 2014;384:164-172.

5. Mamounas EP, Bryant J, Lembersky B, et al. Paclitaxel after doxorubicin plus cyclophosphamide as adjuvant chemotherapy for node-positive breast cancer: results from NSABP B-28. J Clin Oncol 2005;23:3686-3696.

6. Henderson IC, Berry DA, Demetri GD, et al. Improved outcomes from adding sequential Paclitaxel but not from escalating doxorubicin dose in an adjuvant chemotherapy regimen for patients with node-positive primary breast cancer. J Clin Oncol 2003;21:976-983.

7. National Comprehensive Cancer Network. NCCN guidelines breast cancer [Internet]. Plymouth Meeting (PA): NCCN, 2020 [cited 2020 Oct 16]. Available from: https:// www.nccn.org/professionals/physician_gls/default.aspx.

8. Won HS, Kim YS, Kim JS, et al. Clinical outcome and predictive factors for docetaxel and epirubicin neoadjuvant chemotherapy of locally advanced breast cancer. Korean J Intern Med 2020;35:1489-1496.

9. Papadimitriou K, Ardavanis A, Kountourakis P. Neoadjuvant therapy for locally advanced breast cancer: focus on chemotherapy and biological targeted treatments' armamentarium. J Thorac Dis 2010;2:160-170.

10. Hirano A, Shimizu T, Imamura H, et al. The combination of epirubicin plus docetaxel as neoadjuvant chemotherapy in locally-advanced breast cancer. Anticancer Res 2006;26(1B):581-584.

11. Khasraw M, Bell R, Dang C. Epirubicin: is it like doxorubicin in breast cancer? A clinical review. Breast 2012;21:142149. 